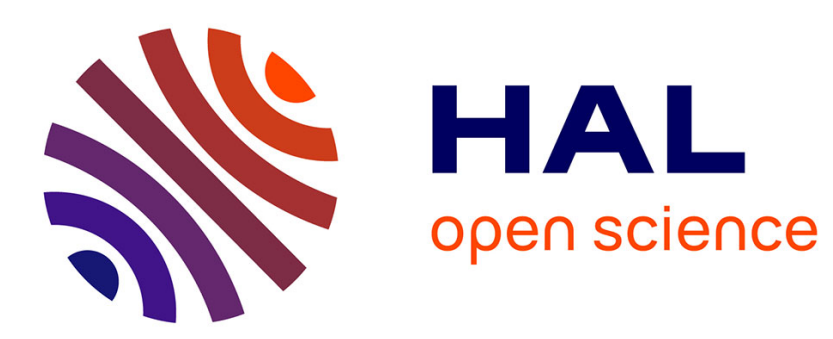

\title{
La correspondance de F.-A. Pouchet avec les membres de l'Académie des Sciences: une réévaluation du débat sur la génération spontanée
}

Dominique Raynaud

\section{- To cite this version:}

Dominique Raynaud. La correspondance de F.-A. Pouchet avec les membres de l'Académie des Sciences: une réévaluation du débat sur la génération spontanée. Archives Européennes de Sociologie / European Journal of Sociology, 1999, 40, pp.257-276. halshs-00006160

\section{HAL Id: halshs-00006160 \\ https://shs.hal.science/halshs-00006160}

Submitted on 25 Nov 2005

HAL is a multi-disciplinary open access archive for the deposit and dissemination of scientific research documents, whether they are published or not. The documents may come from teaching and research institutions in France or abroad, or from public or private research centers.
L'archive ouverte pluridisciplinaire HAL, est destinée au dépôt et à la diffusion de documents scientifiques de niveau recherche, publiés ou non, émanant des établissements d'enseignement et de recherche français ou étrangers, des laboratoires publics ou privés. 
Slightly revised for the European Journal of Sociology, 1999, 40 (2), pp. 257-276.

\title{
La correspondance de F.-A. Pouchet avec les membres de l'Académie des Sciences: une réévaluation du débat sur la génération spontanée
}

\author{
Dominique Raynaud*
}

Résumé. La controverse sur la génération spontanée (1859-1864) qui opposa Pasteur à Pouchet a suscité une large attention de la sociologie des sciences relativiste. Cet article montre que plusieurs comptes rendus ont été biaisés par la version partisane de Pennetier (1907). L'examen de la correspondance entre Pouchet et les membres de l'Académie des Sciences révèle que Pouchet était lié à de nombreux membres de cette institution, et que les deux commissions instituées en 1862 et 1864 n'étaient pas infiltrées par un lobby pastorien. La découverte d'un faux de Pouchet conduit à une réévaluation de cette controverse. La défaite de Pouchet s'explique avant tout par ses retraits répétés devant les commissions, sa croyance dogmatique dans l'hétérogénie, le lobbying de la presse et une recherche démesurée des honneurs. Outre l'absence de réponse à l'argumentation logique développée par ses opposants, une telle transgression des normes de l'ethos scientifique semble avoir été une source d'agacement au sein de l'Académie des Sciences.

Mots-clefs. Controverse, génération spontanée, Pasteur, Pouchet, relativisme, rationalisme.

Summary. The controversy between Pasteur and Pouchet on spontaneous generation (1859-1864) has received great interest in the sociology of scientific knowledge. This paper shows that some statements were shifted by Pennetier's biased version (1907). The study of the correspondence between Pouchet and the members of the Académie des Sciences reveals that Pouchet was on intimate terms with many members of this institution, and also that the two commissions appointed in 1862 and 1864 did not collude with any Pastorian lobby. The discovery of Pouchet's forgery leads to a quite different estimate of this controversy. Pouchet's defeat can be explained by his repeated retreats in front of the commissions, his dogmatic faith in heterogeny, lobbying by the press, and an immoderate need for glory. Beyond the lack of replies to the rationale against heterogeny, such a transgression of the norms of scientific ethos seems to have been a source of irritation among the Academicians.

Keywords. Controversy, spontaneous generation, Pasteur, Pouchet, relativism, rationalism.

La sociologie de la connaissance scientifique [Sociology of Scientific Knowledge] fait grand cas des controverses, sur lesquelles elle montre que les contenus scientifiques sont régulièrement influencés par des facteurs sociaux (politiques, idéologiques ou religieux). Cette thèse a le soutien d'arguments parfois puissants, mais ne parvient toujours pas à faire le consensus de la communauté 
des sociologues et des historiens des sciences. Il faut en voir la raison dans le fait qu'elle se situe précisément au coeur du débat contemporain entre relativisme et rationalismeㄹ.

Parmi les controverses scientifiques qui ont fait l'objet d'une étude de sociologie des sciences, la plus célèbre est sans doute celle qui opposa Louis Pasteur (1822-1895) à Félix-Archimède Pouchet (1800-1872) à propos de la génération spontanée. La question au centre de la discussion était la suivante: peut-on créditer la thèse de la «production d'un être organisé nouveau, dénué de parents, et dont tous les éléments primordiaux ont été tirés de la matière ambiante » (Pouchet, 1859: 1). Les partisans de l'hétérogénie répondaient oui. Leurs adversaires, comme Louis Pasteur, rétorquaient que les micro-organismes qui apparaissaient dans les ballons scellés provenaient de germes qui les avaient contaminés avant qu'ils ne fussent fermés ${ }^{2}$. Ce fut le début d'une puissante controverse qui dura, pour l'essentiel, de 1859 à 1864. La dispute est aujourd'hui si connue dans

*Université Pierre-Mendès-France, BP 47, 38040 Grenoble cedex 9, dominique.raynaud@upmf-grenoble.fr

${ }^{1}$ Pour un rappel des arguments rationalistes et relativistes mobilisés par l'étude des controverses, je renvoie à deux études antérieures dans lesquelles on trouvera le matériel nécessaire à leur évaluation (Raynaud, 1998a, 1998b).

2 Il y aurait beaucoup à dire des aspects scientifiques de la controverse, délaissés dans cet article qui porte exclusiv ement sur ses aspects sociologiques. Quelques observations cependant. La sociologie relativiste aime à rehausser la valeur des expériences de Pouchet. Elles auraient préparé la découverte du Bacillus subtilis résistant à une température de 100 C (Geison, 1981: 372; Latour, 1989: 445; Farley et Geison, 1991: 136). Cette idée est opposable. Primo, après avoir affirmé - a priori - que « l'air qui a traversé l'acide sulfurique est contraire à la génération spontanée » (1859: 252), Pouchet reproduit les expériences de Schulze avec un appareil à cinq boules de Liebig et trouve néanmoins un résultat positif. Le 24e jour, il voit apparaître du Penicillium à la surface du ballon connecté au dispositif, alors que le ballon laissé à l'air libre en est dépourvu [sic] (1859: 272). Secundo, Pasteur devait montrer en janvier 1861 que certains germes restent féconds dans une atmosphère chauffée à moins de $120^{\circ} \mathrm{C}$ alors qu'ils deviennent stériles lorsqu'ils sont portés à une température de $130^{\circ} \mathrm{C}$ pendant 20 à 30 minutes. Tertio, Pouchet parvient à un résultat positif dans des expériences sur de l'air calciné à $150^{\circ} \mathrm{C}$ (Pouchet, 1860). Son disciple Pennetier rapporte même que, devant l'objection faite par Milne-Edwards qu'une température de $100^{\circ} \mathrm{C}$ ne suffit pas à détruire certains germes du foin, Pouchet « porta le corps putrescible à $200^{\circ} \mathrm{C}, 250^{\circ} \mathrm{C}$ et plus, sans entraver l'apparition des proto-organismes [sic] » (1907: 36). Or, aucun micro-organisme ne résiste à la température de coagulation du cytoplasme $\left(120^{\circ} \mathrm{C}\right)$. Les résultats de Pouchet étaient donc dus à une contamination des ballons par des germes extérieurs. En outre, ses talents d'expérimentateur étaient tels qu'il croyait avoir démontré expérimentalement que la lumière rouge favorise l'apparition des micro-organismes animaux; la lumière verte celle des micro-organismes végétaux (1859: 197). Pour le Rouennais, l'hétérogénie était moins un sujet d'expérience qu'une doctrine philosophique. Rappelons à cet égard qu'il admettait les « irrécusables observations de pluies de grenouilles... d'avalanches d'épinoches » comme une preuve flagrante de la génération spontanée (1859: 448). 
les milieux sociologiques qu'on en trouve l'écho dans des publications diverses, et parfois même dans des présentations quelque peu caricaturales. Il s'est en effet progressivement créé un consensus autour de la figure de Pouchet, qui aurait affronté, lors des expertises de l'Académie des Sciences de 1862 et 1864, une coalition des savants liés à Pasteur.

La formation du consensus peut être étudiée en suivant l'altération du récit sociologique de l'épisode crucial que constitue le jugement porté par les commissions sur les travaux de Pasteur et Pouchet. Farley et Geison écrivent: «La commission nommée pour décerner le prix se composait initialement de Geoffroy Saint-Hilaire, Serres, Milne-Edwards, Brongniart et Flourens, mais, avant qu'elle ait pu rendre son jugement, Geoffroy était décédé et Serres avait été exclu ${ }^{3}$ de la liste. Ils furent remplacés par Claude Bernard et Coste et, ainsi, le jury se trouva dès le début composé à l'unanimité de membres défavorables à la génération spontanée » ([1974] 1991: 118). Mendelsohn trace, quant à lui, le portrait suivant de la controverse: « Dans la commission instituée en 1862, tous les membres étaient catholiques ${ }^{4}$, et quelques membres avaient annoncé leur décision avant d'examiner les travaux des concurrents. D'une façon similaire, en 1864, tous les membres étaient unis à l'avance en faveur de Pasteur [...] » (1987: 110). Latour, dans une réinterprétation des données de Farley et Geison, écrit: «Ces commissions ne sont composées que de collègues de Pasteur qui sont tous à peu près convaincus à l'avance » (1989: 435): Pasteur aurait utilisé « ses amis de l'Académie ». Dans un panorama des travaux récents en sociologie des sciences, dressé à l'occasion du colloque d'Amiens, Lécuyer accorde que « L'image de Pasteur sort de cet examen quelque peu modifiée. Il aurait mis ses appuis politiques au service de ses capacités d'expérimentateur » ([1990] 1998: 37). Enfin, dans un commentaire plus récent, Vinck énonce la thèse de la manière suivante: « [L'Académie des Sciences] institua deux commissions composées de membres en faveur de Pasteur; celles-ci accordèrent un soutien inconditionnel et n'eurent que du mépris pour Pouchet » (1995: 114).

\footnotetext{
${ }^{3}$ Précision utile: le naturaliste Pierre Marcel Toussaint de Serres (1783-1862) était alors âgé de 79 ans et devait déc éder la même année. Dans ce contexte, il est probable que l'on doive remplacer les mots « fut exclu » par « se retira ». ${ }^{4}$ L'inexactitude de cette proposition a été démontrée indépendamment par McMullin (1987) et par Crossland (1992: 430). Ce dernier rappelle notamment que Claude Bernard était un « rationaliste laïque » et que Dumas, qui avait entrepris des études sur la fertilisation, s'opposait à la thèse de la génération spontanée surtout pour des motifs scientifiques.
} 
Si l'on suit le récit de la controverse de Farley et Geison à Vinck, on constate immanquablement une érosion progressive des circonstances, puisque l'accord des membres de la commission contre Pouchet est d'abord présenté comme une conséquence de l'agrégation de leur jugement individuel, avant de devenir une finalité orientant la composition de la commission d'expertise. Par ailleurs, aucun de ces comptes rendus n'expose le déroulement fort particulier de ces expertises. La première commission, qui devait décerner le prix Alhumbert à Pasteur le 29 décembre 1862 pour ses expériences sur la génération spontanée, était composée de Claude Bernard, Brongniart, Coste, Flourens et Milne-Edwards. Il faut noter que le prix ne fut pas exactement disputé par Pasteur et Pouchet. En effet, le biologiste rouennais, qui avait déposé ses pièces au concours le 22 septembre (BNF, ms. naf 18111, fol. 116), se retira après une courte entrevue avec MilneEdwards qui lui apprit qu'il estimait devoir accorder son vote à Pasteur « parce que j'ai vu ses expériences et qu'elles m'ont parfaitement convaincu » (Pouchet, 1864: xiv). Comme on sait, Pouchet ne resta pas sur cette défaite, et, secondé par Joly et Musset de la faculté de Toulouse, il entreprit de réfuter les conclusions de Pasteur, par une deuxième série d'expériences qui dureront jusqu'en 1864. Ayant pratiqué de nouvelles observations, Pouchet, Joly et Musset exigèrent de l'Académie des Sciences qu'elle institue une deuxième commission d'expertise pour trancher définitivement la question de la génération spontanée. Composée de Balard, Brongniart, Dumas, Flourens et Milne-Edwards, celle-ci fut votée le 4 janvier 1864 dans la perspective d'une expertise qui devait avoir lieu au printemps. Pouchet et ses collaborateurs demandèrent que les expériences soient reportées au mois de juin, ce que la commission accepta (contre l'avis de Pasteur). En juin, l'équipe de Pouchet refusa de faire ses expériences devant la commission au motif que l'expertise aurait été arrangée par Pasteur. Dans une lettre datée du 18 juin 1864, l'Académie devait laisser aux hétérogénistes une ultime possibilité de présenter leurs expériences. Mais, estimant que la saison favorable était désormais passée, Pouchet et ses collaborateurs se récusèrent une dernière fois. La commission assista donc aux seules expériences de Pasteur et consigna leur exactitude dans le rapport du 20 février 1865. Ainsi, les commissions de l'Académie des Sciences durent se prononcer sur les seuls travaux de Pasteur, parce que Pouchet s'était défaussé à chaque fois. On voit mal comment cette attitude aurait pu lui valoir un jugement favorable.

Il est par ailleurs remarquable que l'érosion progressive des détails de la controverse au profit d'un récit canonique n'a tenu aucun compte des critiques - parfois circonstanciées, parfois bru- 
tales - qui ont été adressées depuis lors à cette lecture relativiste de la controverse entre Pasteur et Pouchet (Roll-Hansen, 1979; McMullin, 1987; Gálvez, 1988; Bunge, 1992; Crosland, 1992; Darmon, 1995). Nous sommes en présence de deux lignes indépendantes d'« interprétation » de la controverse et un consensus aussi fragile suffit à demander une réouverture du dossier. L'objectif de cet article pourrait être d'établir les rapports exacts entre Pouchet et les membres de l'Académie des Sciences à partir d'une étude de sa correspondance. Celle-ci est archivée:

1) au Muséum d'Histoire naturelle de Rouen ${ }^{5}$ (cartons FAP 30 [3012: Bernard, 3014: Blai nville, 3047: Coste, 3078: Flourens], FAP 31 [3102: Geoffroy Saint-Hilaire, 3176: Pasteur, 3192: Rayer], et FAP 51, 52, 53).

2) à la Bibliothèque municipale de Rouen (Autographes normands, mss. g 182, g 185, m 3690, p 55, ainsi que le carton non catalogué du Don Levillain, portant le $n^{\circ}$ d'inventaire 1754).

3) à la Bibliothèque nationale de France (mss. naf 3305, 18096, 18106, et naf 18111, mal catalogué, étant accessible à partir des «Lettres reçues par Flourens», au lieu des « Lettres émises par Pouchet $\left.»^{6}\right)$.

Dans la suite de cet article, les pièces seront citées: MHR (Muséum d'Histoire naturelle de Rouen), BMR (Bibliothèque municipale de Rouen), BNF (Bibliothèque nationale de France).

\section{Pouchet et les membres de l'Académie des Sciences}

Le dépouillement de ces archives montre tout d'abord que Pouchet a entretenu une correspondance suivie avec les membres de l'Académie des Sciences, et parfois en des termes très amicaux. Ce fait, qui brise quelque peu l'image d'un Pouchet exilé dans sa province et éloigné du Tout-Paris où se prennent les décisions importantes, s'explique d'abord par le fait qu'il était correspondant de l'Académie des Sciences depuis vingt ans à l'époque de la controverse (Pouchet fut

\footnotetext{
${ }^{5}$ J'exprime ici toute ma gratitude à Mme Monique Fouray, Conservateur du Muséum d'Histoire naturelle de Rouen, qui a grandement facilité mes recherches sur ces documents.

${ }^{6}$ Cette collection de 524 fols. correspond exactement à la correspondance mise en vente chez Durtal, 12, rue Jacob à Paris, en 1936: «Correspondance échangée entre F.-A. Pouchet, de Rouen, et N. Joly, de Toulouse, pendant les années 1861 à 1867, où se trouvent décrites et discutées en détail les célèbres expériences qui opposèrent ces deux savants à Pasteur [...] Autographes de Musset, Flourens, V. Meunier, etc. » (Lambert, 1936: 39).
} 
élu à la majorité le 17 décembre 1849). Vingt ans, durant lesquels le naturaliste rouennais eut l'occasion de fréquenter ses pairs, à commencer par Pierre Flourens (1794-1867), physiologiste et secrétaire perpétuel de l'Académie des Sciences de 1833 à 1868.

Flourens avait entendu parler de Pouchet bien avant que ne débute la controverse sur la génération spontanée. Il semble avoir apprécié les travaux sur la reproduction du naturaliste rouennais: «Je remettrai mardi à l'Académie l'exemplaire qui lui est destiné, et je tâcherai de le faire en des termes qui répondent à mon estime pour le livre et pour l'auteur » (4 février 1847, MHR, FAP 3078); « Croyez bien que je n'oublierai pas votre bel ouvrage; je vais m'en occuper, et j'en parlerai à M. Vitet. Comptez sur le véritable attachement de votre dévoué confrère » (22 avril [1849], MHR, FAP 3078). Pierre Flourens termine une autre de ses lettres par la formule: «Considération très distinguée et toute mon affection »(17 avril 1853, MHR, FAP 3078). L'étroitesse des rapports avec le secrétaire perpétuel sont également attestées par Pouchet qui écrit à son ami Noël: «G. Flourens, que j'ai vu élever, que son père recommandait à mon affection $[\ldots]$ » (sans date, BMR, ms. $\mathrm{n}^{\circ}$ inventaire 1754). Les relations de Pouchet avec Pierre Flourens étaient assez intimes pour avoir connu son fils Gustave. Quand on sait que Flourens fit partie des deux commissions chargées de juger le différent avec Pasteur, il est plus difficile d'accepter le compte-rendu habituel de la controverse: si la voix de Flourens fut acquise à Pasteur, c'est sur la base d'arguments scientifiques, et non par suite de son appartenance à quelque coterie pastorienne.

Victor Coste (1807-1873) est l'un des académiciens avec qui Pouchet fut très lié. Les deux hommes partageaient le même intérêt pour les problèmes piscicoles. Cet embryologiste était Professeur au Collège de France et membre de l'Institut. Le Muséum d'histoire naturelle de Rouen conserve une quinzaine de lettres de Victor Coste. Pouchet, dont les formules finales ne laissent pas d'ambiguïté sur la nature amicale de leurs relations: « Mille compliments affectueux » (1er février 1854, MHR, FAP 3047); "Mille amitiés » (14 novembre 1855, ibidem); « Tout à vous de coeur » (sans date, ibidem). Et, après le débat sur la génération spontanée, cette fois: «Vieilles amitiés » (27 octobre 1869, ibidem). On peut en outre estimer la considération de Victor Coste envers Félix-Archimède Pouchet, à la mesure de la proposition qu'il lui fit dans une de ses lettres. Coste se proposait en effet de devenir le collaborateur du rouennais pour étudier la reproduction des poissons: «Je serai d'autant plus disposé à vous seconder dans cette entreprise, que ce serait 
pour moi un nouveau champ d'observation où j'aurais grand plaisir à être votre collaborateur [...] Croyez à mes plus vives sympathies » (17 juin 1854, MHR, FAP 3047). Quand on sait que Coste faisait partie de la commission de l'Académie des Sciences chargée de décerner le prix Alhumbert, on a peine a croire que l'homme donna sa voix à Pasteur parce qu'il faisait partie d'un lobby pastorien.

Même si Milne-Edwards et Balard — dont Pasteur fut l'étudiant — étaient des adversaires de l'hétérogénie, il est peu crédible d'affirmer que les commissions d'expertise des travaux sur la génération spontanée étaient a priori acquises à Pasteur. Ni Flourens, ni Coste, ni Brongniart lequel était botaniste — ne rentrent dans le cercle des Pastoriens ${ }^{7}$. Ainsi les commissions avaientelles été composées d'une manière équilibrée - si tant est que l'on puisse suspecter une influence des relations amicales sur le jugement scientifique auquel devait parvenir la commission.

On se doit d'ajouter que Pouchet était en relation étroite avec d'autres membres de l'Institut. Il avait dédié son Hétérogénie à Pierre Rayer (1793-1867), « en hommage public de sa reconnaissance » (1859: x). Rayer lui vouait en retour des relations d'estime qui durèrent bien après la

\footnotetext{
${ }^{7}$ L'étude parallèle de la correspondance de Pasteur montre que les seules relations que Pasteur ait eues à l'Académie étaient: Balard, dont Pasteur avait été l'étudiant, Dumas et Biot (lequel mourut au début de 1862, sans siéger à la commission du prix Alhumbert). Pasteur entretenait avec Flourens, Chevreul, Serres ou Bernard des relations entachées d'un certain formalisme. Par ailleurs, la chronologie permet d'affirmer que les amitiés supposées de Pasteur n'ont pas interféré avec le déroulement de la controverse. L'idée d'une intervention personnelle de l'Empereur dans cette affaire n'a jamais été démontrée. Il existe d'autres indices qu'il n'y eut pas de collusion entre les questions scientifiques et les relations avec le colonel Favé, ordonnance de l'Empereur. L'épisode suivant en témoigne. Le colonel Favé, polytechnicien, briguait un siège à l'Académie des Sciences. S'en étant ouvert à Pasteur, il reçut la réponse suivante: «J'étais loin de prévoir, Monsieur, que je serais parmi ceux qui ne contribueraient pas à votre succès [...] Je ne vois pas le moyen de ne pas voter pour M. Foucault » (1951: 161). Jean-Bernard-Léon Foucault (1819-1868) fut élu les mois suivants, et Favé dut attendre 1876 pour trouver une occasion favorable. Cette réponse de Pasteur est datée du 3 avril 1864: c'est-à-dire avant que la commission instituée par l'Académie des Sciences ne se prononce sur les expériences contradictoires de Pasteur et Pouchet. Si Pasteur et Favé avaient été liés par des relations d'assujettissement, de deux choses l'une: 1) soit Pasteur aurait voté pour lui; 2) soit Favé aurait nourri une telle rancoeur à l'égard de Pasteur, qu'il aurait fait retourner le jugement de la commission au bénéfice de Pouchet. Aucune de ces deux choses ne s'est bien évidemment produite. Enfin, on observera que tous les faits concrets censés établir les rapports entre Pasteur et l'Empereur sont habilement renvoyés par Farley et Geison (1991: 126) aux « années 1860 ». Or, Pasteur fut reçu au Palais de Compiègne en 1865; ses études sur le vin et le ver à soie, financées par Napoléon III, datent de 1866 et 1870 ; il fut
} 
controverse. Il lui écrit le 29 novembre 1869: «Je me suis empressé d'offrir à la Société de Biologie vos nouvelles études sur la génération spontanée [...] Pour moi, je suis avec intérêt ces études si difficiles et si délicates et je m'empresse de vous adresser tous mes remerciements » (MHR, FAP 3192). Par ailleurs, on sait, par le témoignage même de Pouchet, que lorsqu'il s'installa à Paris pour faire une première série d'expériences devant des membres de l'Académie, Serres l'invita à utiliser son matériel. Le Rouennais garde un souvenir ému de celui qui, « avec une obligeance que n'oublierai jamais, avait mis son laboratoire à ma disposition » (1864: xv). Enfin, Pouchet était un intime d'Isidore Geoffroy Saint-Hilaire (1805-1861), avec qui il avait fondé la Société zoologique d'Acclimatation, et dont il connaissait le père de longue date ${ }^{8}$. Isidore Geoffroy SaintHilaire était, à l'instar de son père, membre de l'Académie des Sciences (depuis 1833). L'attitude bienveillante de Serres et de Geoffroy Saint-Hilaire est incidemment confirmée par Pouchet. Il écrit à Joly de Toulouse: «Vous avez là un excellent et loyal ami en M. Geoffroy Saint-Hilaire, justice vous sera faite. Lui n'a aucun parti pris. Ni M. Serres »; «M. Serres, le seul que j'ai pu rencontrer, ne paraît nullement hostile à nos travaux et plutôt disposé à les appuyer » (BNF, naf 18111, fol. 47, 113).

Si le jugement sur la génération spontanée avait été "préparé », comme le prétend la sociologie des sciences relativiste, les proches de Pouchet à l'Académie des Sciences n'auraient pas manqué: 1) de défendre au sein de l'Académie les principes d'équité et d'impartialité contre les assauts, jugés illégitimes, de Pasteur; 2) de relater à leur ami rouennais les fait et gestes relatifs à une manipulation des commissions. Or ni Flourens - secrétaire perpétuel de l'Académie, rappelons-le - ni Coste, ni Rayer, ni Serres, ni Isidore Geoffroy Saint-Hilaire n'ont engagé de telles actions.

nommé commandeur de la Légion d'Honneur en 1868. L'influence éventuelle des idées du Second Empire concerne des faits qui sont tous postérieurs au règlement de la controverse (1864).

${ }^{8}$ Le naturaliste Étienne Geoffroy Saint-Hilaire (1772-1844) était professeur au Muséum d'histoire naturelle de Paris, de même que Henry Ducrotay de Blainville (1777-1850), avec qui il partageait d'ailleurs une hostilité avouée à l'égard des thèses de Cuvier. En 1827, Blainville avait offert à Félix-Archimède Pouchet une place de préparateur au Muséum. C'est sans doute par sa filiation à Geoffroy Saint-Hilaire et à Blainville que le Muséum de Paris proposa, en 1855, un poste de professeur à Pouchet en remplacement de Duvernoy (poste qui devait finalement lui échapper). Blainville fut également un membre influent de l'Académie des Sciences. 


\section{Une lettre de Geoffroy Saint-Hilaire}

Dans toute la correspondance reçue par Pouchet, se distingue une lettre d'Isidore Geoffroy Saint-Hilaire. Cette lettre est importante car elle laisse entendre que le prix Alhumbert de 1862 avait été créé pour Pouchet. Cette lettre, datée du 31 août 1859, est conservée au Muséum d'Histoire naturelle de Rouen (FAP 3102). L'auteur commence par remercier Pouchet pour son Hétérogénie, dont il vient d'entreprendre la lecture.

\section{Expertise en écriture}

La lettre du 31 août 1859 adressée à Pasteur est rédigée dans une écriture régulière. Elle n'est pas de la main de Geoffroy Saint-Hilaire, comme on peut le constater en la rapprochant d'un autographe d'Isidore Geoffroy Saint-Hilaire.

Un deuxième sondage, centré sur la correspondance de Félix-Archimède Pouchet, révèle une similitude entre cette écriture manuscrite et certains courriers officiels émanant du Muséum d'Histoire naturelle de Rouen. Compte tenu du fait que cette écriture appartient à une personne de l'entourage de Pouchet, on peut admettre que la lettre du 31 août 1859 est la copie d'un original perdu ou endommagé. Mais par qui a-t-elle été recopiée? On peut ici avancer deux hypothèses: 1) cette lettre a été écrite par Pouchet; 2) cette lettre a été rédigée par un secrétaire, un collaborateur ou un proche parent du biologiste rouennais.

La première hypothèse semble devoir être infirmée par une comparaison de la lettre d'Isidore Geoffroy Saint-Hilaire avec un autographe de Félix-Archimède Pouchet (Planches 1 et 4). Toutefois, une analyse plus serrée dévoile que la lettre de Geoffroy Saint-Hilaire n'est pas rédigée dans une écriture aussi régulière qu'il y paraît. On note l'existence de variantes dans le script de certaines lettres. On peut y distinguer une écriture contrôlée - «typique » — dont est composé l'essentiel du texte, et une écriture relâchée — « atypique » — qui apparaît dans quelques occurrences de certaines lettres. Si l'on compare les minuscules $[\mathrm{d}, \mathrm{f}, \mathrm{j}, \mathrm{p}, \mathrm{t}, \mathrm{x}]$ et les majuscules $[\mathrm{C}, \mathrm{E}$, $\mathrm{P}, \mathrm{Q}]$ de l'écriture atypique avec les caractères correspondants d'un autographe de Pouchet, on 
est amené à conclure qu'il s'agit d'une seule et même écriture ${ }^{9}$. C'est donc Pouchet qui a rédigé la lettre qu'Isidore Geoffroy Saint-Hilaire lui aurait adressée le 31 août 1859. Il reste maintenant à déterminer s'il s'agit d'une copie scrupuleuse ou d'un faux, question devant laquelle l'expertise en écriture reste muette.

\section{Examen stylistique}

Lorsqu'on examine attentivement le style de cette lettre, on est frappé par certaines tournures qu'on ne retrouve dans aucune des lettres de Geoffroy Saint-Hilaire. L'étude systématique d'un échantillon de seize correspondances, dont la plupart de trois ou quatre pages (MHR, FAP 3102) renseigne sur les formes stylistiques caractéristiques de l'auteur. Les lettres de Geoffroy Saint-Hilaire sont directes, quoique non dépourvues de finesse. L'expression y procède toujours par touches précises et ne s'accompagne jamais de très longs développements.

De fait, certaines tournures de la lettre du 31 août 1859 paraissent inhabituelles sous la plume de Geoffroy Saint-Hilaire. Les passages douteux, du point de vue de l'authenticité stylistique, ont été soulignés. Voici le contenu de cette lettre:

Paris, le 31 août 1859

Mon cher et très-honoré Confrère,

J'ai éprouvé le plus grand regret de m'être trouvé absent lorsque vous avez eu l'extrême amabilité de m'apporter vous-même l'ouvrage dans lequel vous reprenez, de fond en comble, la grande question de l'hétérogénie. Quoique Rouen et Paris se touchent presque, aujourd'hui, les occasions de vous voir sont rares: chacun de nous, retenu dans son cabinet, y vit pour la science, et lui sacrifie tout, même jusqu'aux jouissances des relations amicales et confraternelles.

Au moins aurais-je voulu vous remercier aussitôt par écrit. Mais j'étais accablé d'épreuves, dont la correction suspendait un départ arrêté pour le 25, et qui n'est pas encore effectué. Une des compensations que j'aurais éprouvées, pour la perte d'une semaine de mes très-courtes vacances, est le plaisir de vous lire. C'est à votre livre [2] que j'ai donné tous les moments que je pouvais enlever à mes travaux, rendus urgents par la nécessité de publier mon demi volume avant mon départ, ou du moins de l'avancer tellement que je n'aie plus à m'occuper à mon retour que de la suite.

\footnotetext{
${ }^{9}$ Les lettres manuscrites et le tableau comparatif des écritures d'Isidore Geoffroy Saint-Hilaire et de Félix-Archimède Pouchet n'ont pu, pour des raisons techniques, être reproduites ici, désormais: D. Raynaud, Sociologie des controverses scientifiques, Paris, PUF, 2003, pp. 65-67.
} 
C'est ce qui fait que je suis encore peu avancé dans la lecture de votre ouvrage. Je viens de terminer ce matin la métaphysique. J'avais lu hier la partie historique.

Je crois qu'on ne pouvait mieux poser, que par ces deux articles si savants et si philosophiques, la question que vous traitez; ce sont les préliminaires les plus propres à la poser dans toute sa grandeur, et à la dégager de tous les éléments étrangers dont on l'a compliquée, au moins autant qu'il est possible, car vous ne parviendrez jamais à empêcher qu'une foule de personnes (qui ne sont pas toujours les plus religieuses en réalité!) vous opposent les arguments théologiques, malgré la démonstration que vous donnez de la liberté laissée ici à la science [3] par la théologie. - La science a d'ailleurs ses voies propres, et elle a le droit d'y marcher sans se préoccuper d'autre chose que de la recherche de la vérité. - Mais on ne le comprend pas encore assez, et il y a, dans le choix du sujet que vous avez pris, autant de courage à ce point de vue, qu'il vous en faut d'ailleurs pour lutter contre les difficultés propres du sujet.

Vos appréciations scientifiques m'ont paru parfaitement justes. Vous jugez bien vos devanciers, vous faites bien ressortir en quoi ils ont avancé, arrêté ou fait dévier la science. J'ai vu en particulier, avec une grande satisfaction, la justice que vous rendez à Lamarck, si injustement traité durant sa vie et après sa mort: je me propose de faire parvenir ce que vous dites à la connaissance de son fils, inspecteur général des Ponts et Chaussées, qui est venu souvent à mes cours, et m'a adressé souvent des lettres relatives, soit à mes sympathies pour les travaux de son père, soit à mes dissidences sur divers points. [4] Le seul de vos devanciers envers lequel je crois qu'il y aurait un peu à réclamer, c'est Bonnet. Je suis loin d'en partager les vues générales; mais que de belles et ingénieuses idées émises à l'appui d'erreurs que je n'appellerai pas belles, car il n'y a de beau que le vrai, mais qui sont d'ingénieuses et brillantes conceptions dont peu d'esprits sont capables.

Je reprendrai ce soir et demain matin votre livre. J'ai hâte d'arriver à vos expériences. Fe n'ai pas ici de parti pris. Je crois ce que vous me dites de la puissance créatrice continuée dans le temps, très sage, très élevé et, dans le vrai sens, très religieux. La génération spontanée est, comme vous le dites très-bien, rejetée par la plupart sur l'autorité du maître. Or, en science, l'autorité du maître est un pauvre argument; car l'élève sait ce qu'a su le maître, et nécessairement quelque chose de plus. Venons donc au fait, à l'expérience; ce sont là les vrais maîtres. Aussi ai-je beaucoup contribué à faire admettre la question de prix proposée par l'Académie et dont le choix a dû vous satisfaire et vous flatter; car, avant vos communications à l'Académie, qui eût pensé, qui eût osé lui proposer un tel sujet? C'est à M. Flourens que revient le mérite de l'avoir mis en avant; mais je puis me rendre cette justice que son adoption m'est due; car nos trois autres collègues, par conséquent la majorité, ne voulaient pas qu'on parût douter, et M. Flourens qui n'aime pas beaucoup la lutte, allait laisser tomber sa proposition. Seulement il a fallu concéder une rédaction qui n'engageât nullement l'Académie; et ici, il y avait d'ailleurs sagesse à ne pas s'engager. Cette question, en effet, a des profondeurs immenses, et plus on y pénètre, et plus on s'en convainc. La distinction que vous faites à la fin de votre partie métaphysique me semble difficile elle-même à admettre d'une manière absolue. Temporairement, pour le présent, et pour longtemps encore, oui. Mais ensuite? Qui le sait. Vous entreprenez de démontrer qu'avec des éléments organiques, on peut créer des êtres organisés, et il est certain [6] qu'il y aurait folie à vouloir aller au-delà aujourd'hui. Mais pourra-t-on arriver un jour à créer des éléments organiques, ou pour parler ici la langue chimique, des principes immédiats de toute pièce? Vous savez ce qu'ont déjà fait dans cette direction quelques chimistes, principalement Mr Berthelot et vous semblez traiter la première et la 
seconde partie de la même question, et si vous avez tous deux raison, il s'en faudra de bien peu que Lamarck n'ait eu raison d'être aussi audacieux.

En voilà bien long, cher Collègue et ami, et cependant je ne terminerai pas sans vous dire combien je suis reconnaissant de la belle justice que vous rendez à mon père, et vos trop bienveillantes paroles envers moi. J'y vois une preuve de votre amitié plus que de votre justice, et si elles me sont moins flatteuses, ainsi comprises, elles m'en sont plus chères. - Je veux vous dire aussi que j'ai lu votre préface avec un sentiment de sympathie bien [7] sincère; vous y parlez des uns pour les remercier; vous vous taisez sur d'autres dont vous aviez le droit de vous plaindre: cette modération vous honore, et en vous élevant d'autant plus au-dessus de telle injustice, que nous n'avons pas oublié, vous montrez d'autant mieux combien elle a été grande et reste injustifiable.

Adieu, agréez l'expression de mes sentiments très-distingués et dévoués.

I. Geoffroy St Hilaire.

On observe dans cette lettre, outre des développements fastidieux peu conformes à l'esprit de Geoffroy Saint-Hilaire: 1) l'usage de superlatifs et d'hyperboles qui donnent un ton grandiloquent à la pensée; 2) une référence constante à la conviction de l'homme de science; 3) des accents positivistes étrangers à l'auteur; 4) une apologie des héros victimes de l'injustice - image que Pouchet n'a cessé de répandre sur son compte à partir des années 1855 (année de son échec au poste de professeur qui s'était ouvert au Muséum de Paris). Or, ces quatre traits stylistiques, étrangers à la pensée de Geoffroy Saint-Hilaire, sont la marque de diverses publications et lettres de Pouchet. On peut en faire la démonstration systématique:

1) les superlatifs de la lettre du 31 août 1859 («articles si savants et si philosophiques; très sage, très élevé et très religieux ») émaillent de nombreux textes du Rouennais. Dans ses lettres à Joly, son collaborateur toulousain, on lit: « d'une si extraordinaire manière; les si drôles de faits; un si magnifique appui »(BNF, naf 18111, fol. 8, 13, 18 et 21). On retrouve les mêmes formules dans L'Hétérogénie: « La si dédaigneuse réprobation; une armée plus magnanime et plus disciplinée » (1859: 5).

2) la ténacité du savant, dans la lettre du 31 août 1859 («vit pour la science, lui sacrifie tout; la science a ses voies propres, et elle a le droit d'y marcher sans se préoccuper d'autre chose que de la recherche de la vérité; ceux qui ont fait dévier la science ») est typique de la plume de Pouchet. Il écrit à Joly: « Courage, donc, et marchez en avant, tant que nous aurons la conviction d'être dans le vrai; Courage, noble ami, il ne faut pas se laisser abattre; Je ne cesserai jamais la 
bataille »(BNF, naf 18111, fol. 21, 50, 77 et 123).

3) les accents positivistes de la lettre de Geoffroy Saint-Hilaire («Je n'ai pas de parti pris; rejeter l'autorité du maître; l'expérience, ce sont là les vrais maîtres ») font également partie de la rhétorique de Pouchet. En atteste sa correspondance avec Pasteur: « La chimie, quelque puissante qu'elle soit, jamais n'anéantira de tels faits; quand il s'agit d'observations délicates, je n'affirme les chose qu'après le plus sérieux examen » (BNF, naf 18106, fol. 47, 49). Dans la préface de L'Hétérogénie, Pouchet nous avertit que le livre se compose de deux sections: « la partie expérimentale, qui est la seule fondamentale, et la partie théorique, qui n'en forme qu'un fragment accessoire » (1859: vii).

4) l'apologie des victimes de l'injustice, qui ponctue la lettre non autographe (« La justice que vous rendez; si injustement traité; la belle justice que vous rendez; au-dessus de telle injustice ») affecte sa correspondance avec Joly: "Justice vous sera faite », lui écrit-il (BNF, naf 18111, fol. 47). Pouchet se plaint aussi, dans une lettre de 1864, de toutes « ces distinctions dont M. le Ministre honore tant de ses nullités universitaires; il accable de toutes ses faveurs le seul petit coin de Paris ». Dans son Histoire des sciences naturelles, Félix-Archimède Pouchet écrivait déjà avec la même conviction: « Nous venons défendre le Moyen Âge des injustes reproches dont il a souvent été l'objet » (1853: 7).

Répétons-le, cette rhétorique de la grandeur et du combat contre l'injustice est totalement étrangère aux seize lettres d'Isidore Geoffroy Saint-Hilaire examinées (MHR, FAP 3102). L'analyse stylistique permet donc d'établir que Pouchet a manipulé le contenu de la lettre de Geoffroy Saint-Hilaire en laissant des indices de son oeuvre. Mais, à l'opposé, la lettre contient des détails qui ne peuvent pas être sortis de l'imagination de Pouchet: son correspondant s'y dit accablé par la correction d'épreuves, il envisage de communiquer L'Hétérogénie au fils Lamarck, un habitué de ses cours, regrette que Bonnet ne soit pas cité, etc. Ces indices prouvent que Pouchet n'a pas entièrement composé la lettre. On peut alors conclure que Pouchet aura reçu une lettre d'Isidore Geoffroy Saint-Hilaire, mais qu'il en aura manipulé le contenu à son avantage. Quelle que soit l'ampleur de ces transformations, cette lettre est un faux. 


\section{L'ethos de la science}

Il convient maintenant d'évaluer les conséquences de cette découverte sur le débat de la génération spontanée, et, surtout, de voir comment elle peut affecter la démonstration sociologique que certains en ont tiré. Fort paradoxalement - puisque la nouvelle sociologie des sciences a l'intention de s'émanciper de l'approche mertonienne - cette lettre a pour effet de recentrer l'attention sur l'« ethos de la science », aspect très souvent négligé dans l'étude de la controverse entre Pasteur et Pouchet.

\section{La notion d'ethos scientifique}

Merton donne de l'ethos la définition suivante: «L'ethos de la science est le complexe de valeurs et de normes teintées d'affectivité [affectively toned complex], auxquelles l'homme de science est tenu de se conformer » (1973: 268-269). Il expose à la suite quatre normes: universalisme, communisme (au sens strict d'une mise en commun des connaissances), désintéressement et scepticisme organisé (1973: 267-278). Ce schéma a ensuite été complété par l'adjonction de deux nouvelles normes: priorité et humilité (1973: 286-324). Ces normes de l'ethos scientifique furent massivement abandonnées par la sociologie des sciences à la suite de la critique radicale faite par Barnes et Dolby (1970) et quelques autres comme Norton (1978), qui considéraient que les normes mertoniennes offraient une image idéalisée et quelque peu caricaturale de la science. Ces auteurs sont parvenus à indexer des comportements déviants sur à peu près chacune des normes de l'ethos scientifique: les pratiques effectives de la science seraient donc indissociables de biais idéologiques, du secret, de la fraude scientifique ou de l'attachement aux idées.

Cette critique est à la fois fondée et contestable. Fondée, parce que les comportements qui sont présentés sont bien déviants. Contestable, parce que l'idée d'opposer des « contre exemples » à des normes de comportement dévoile une bizarrerie d'interprétation de la notion de « norme ». Qui penserait à opposer des contre exemples au fait que le meurtre, l'inceste ou l'adultère soient condamnés dans l'Ancien Testament? De telles interdictions attestent au contraire que de tels comportements existaient dans l'Antiquité. Il en va de même des normes de l'ethos scientifique; et Merton lui-même ne manque pas de souligner que l'histoire des sciences est ponctuée de fraudes et de plagiats (1973: 309-312, 312-316, 397-398). Ge que signifie le sociologue américain par le 
terme de «normes », c'est que tout comportement conforme à la norme est valorisé par les membres de la communauté scientifique, alors qu'un comportement déviant est réprouvé au titre de transgression ${ }^{10}$. Le critère pour établir l'inexistence d'une norme n'est pas d'opposer un compo rtement déviant, mais de montrer que tel comportement déviant connu n'a pas été sanctionné par la communauté. C'est d'ailleurs le canevas même que suit Merton dans l'analyse fonctionnelle du système des referees vis-à-vis des plagiats (1973: 492).

Cette mise au point sur la notion d'ethos scientifique montre: 1) que la notion conserve sa validité, en dépit du fait qu'elle ait été reléguée par la sociologie des sciences d'obédience relativiste. Le fait est justement souligné par Ben-David (1991: 451-468); 2) que l'appareil mertonien offre un cadre sociologique adéquat pour interpréter la fraude scientifique. Il convient alors d'en appliquer le principe à l'attitude de Pouchet dans le débat sur la génération spontanée.

\section{L'attitude scientifique de Pouchet}

Le contenu de la lettre falsifiée par Pouchet nous informe sur la forte détermination de son auteur à remporter une victoire dans le débat qui l'opposait à Pasteur. Car enfin, ce que nous apprennent les parties les moins authentiques de cette lettre, c'est:

1) que Pouchet aurait dû être admiré de ses pairs pour s'être attaqué à une question qui «a des profondeurs immenses ». Qu'ils auraient dû, à l'instar de Geoffroy Saint-Hilaire qui n'avait pas de « parti pris », estimer que son appréciation scientifique était « juste » et qu'il y avait « folie à aller au-delà ».

2) que l'idée du prix Alhumbert consacré à la génération spontanée était due aux recherches

\footnotetext{
10 Nous en restons ici à une interprétation classique des normes. Notons toutefois que la notion de norme, général ement perçue dans un cadre holiste (une communauté imposant d'en-haut des comportements-types à des individus) est aisément reconstructible dans le cadre de l'individualisme méthodologique. Le communisme peut ainsi être saisi comme la cristallisation, dans une communauté scientifique, d'un principe que ses membres actuels ont encore de bonnes raisons de promouvoir. Si tous les producteurs de connaissance gardaient jalousement leurs résultats, aucun ne pourrait s'appuyer sur les expériences d'autrui. Il en résulterait que: 1) chacun perdrait un temps infini à établir les théories d'observation dont il a besoin pour réaliser ses propres expériences; 2) personne ne serait à l'abri de redécouvertes inutiles de résultats établis depuis fort longtemps (mais toujours inconnus); 3) tous seraient à la merci de leurs lubies ou de leurs erreurs de jugement, aucune activité critique ne pouvant s'exercer dans cette pseudo-communauté.
} 
et aux communications de Pouchet à l'Académie des Sciences, avant lesquelles il eût été proprement « impensable de proposer un tel sujet ». Ce fait est inexact. Gálvez (1988: 348-351) a montré que le prix Alhumbert de 1862 s'inscrivait dans la continuité des questions proposées au concours depuis les années 1840.

Que tirer de [1+2], sinon que la commission de l'Académie des Sciences aurait du décerner le prix Alhumbert à Pouchet, prix qui était en quelque sorte taillé à sa mesure? Par la conclusion implicite qu'il appelle, ce faux constitue une pierre d'angle du débat sur la génération spontanée, parce qu'il nous conduit à porter un jugement rétroactif peu complaisant sur les décisions prises par les membres de l'Académie des Sciences à l'égard de Pouchet. Bien évidemment, le fait que le document qui nous conduit à tirer cette conclusion soit un faux, nous amène à réviser notre jugement, tant sur les conditions d'attribution du prix Alhumbert que sur la compassion que l'on pouvait éprouver pour l'adversaire de Pasteur. Deux des normes mertoniennes (scepticisme organisé, priorité) ont manifestement été transgressées par Pouchet.

a) scepticisme organisé. Selon le point de vue mertonien, le scepticisme n'est pas un trait personnel de certains chercheurs, mais plutôt l'effet d'une obligation institutionnelle [institutional mandate]. Toutefois, un savant peut délibérément enfreindre cette norme par une attitude relevant davantage de la conviction que de la neutralité qui doit accompagner la production des connaissances. Le comportement de Pouchet est clairement déviant à cet égard. Le naturaliste rouennais écrit à Noël: «Je puis vous assurer que ['hétérogénie] va triompher, dans un temps prochain, de tous les obstacles ». Car, alors que la controverse n'en est qu'à son début, « Pasteur a été écrasé » (BMR, ms $n^{\circ}$ inventaire 1754). Pouchet emploie, dans les années 1860-1862, un registre similaire avec son collaborateur toulousain: «Le savant chimiste [Pasteur] s'est suicidé ». "Je le combats à la face du ciel pour notre cause commune ». «Notre cause est absolument gagnée ». Puis, devant les difficultés qui commencent à surgir, Pouchet éprouve le besoin de galvaniser ses troupes: « L'un de nous seul, on l'eût étouffé; trois, nous formons un faisceau terrible et indestructible ». Il répète à Joly: «Vous et moi qui travaillons si courageusement à une sainte cause »; il faut « combattre pour notre sainte cause ». « Me voici à Paris, m'occupant de notre sainte cause ». Avant d'avouer finalement: «Nous sommes invincibles » (BNF, naf 18111, fol. 17, 21, 26, 30, 46, 52, 77, souligné 
dans l'original). Cet échantillon suffit à montrer que le rapport de Pouchet à l'hétérogénie échappe à tout scepticisme. La génération spontanée était pour lui un dogme, auquel il fallait assurer un triomphe sans partage. On sait ce qu'il en fut.

b) priorité. Dans les textes de Merton, la norme de priorité, qui garantit au chercheur d'être crédité d'une découverte s'il l'effectue avant ses pairs, induit des transgressions dont les principales se caractérisent par un culte déplacé de l'originalité. En voulant être premier à tout prix, un chercheur peut être conduit à imaginer des moyens illicites pour parvenir à une fin qu'il sait ne pas pouvoir atteindre par des moyens réguliers. C'est alors que se produit la fraude ou le plagiat. Ces deux cas sont examinés par Merton dans le chapitre sur les « types de réponse à l'importance culturelle de l'originalité » (1973: 309-316). Après avoir rappelé que Charles Babbage avait déjà entrepris une classification des fraudes scientifiques, Merton donne plusieurs exemples d'affaire célèbres. On sait qu'en France, au XIXe siècle, Vrains-Lucas confectionna de nombreux faux afin de les revendre à des savants trop crédules, tel le mathématicien Chasles. L'affaire VrainsLucas est intéressante parce qu'elle stigmatise le credo fondamental du faussaire. Dans une correspondance imaginaire entre Pascal et Newton, Vrains-Lucas fait dire au jeune Newton: «Tout homme qui n'aspire pas à se faire un nom n'exécutera jamais rien de grand » (1973: 310). Cette maxime caractérise assez bien l'attitude de Pouchet vis-à-vis de l'hétérogénie. Dans certaines correspondances, Pouchet fait des promesses qui relèvent explicitement d'une recherche de gratifications personnelles. Dès le 4 novembre 1860, il écrit à Joly de Toulouse: "Je crois que [notre noble cause] est de nature, par son grand retentissement, à jeter plus de gloire sur nos noms que tout autre sujet » (BNF, naf 18111, fol. 17, souligné dans l'original). Au début de l'année 1862, Joly paraît affecté par le retrait de Pouchet devant la commission du prix Alhumbert. Il aurait eu l'intention d'abandonner sa collaboration avec Pouchet. Le rouennais lui envoie alors, le 2 février 1862, une lettre visant à le remotiver: «De quoi vous plaignez-vous, sapristi! [...] On parle de nous sur les déserts rivages de l'Afrique. Je ne ris pas. On s'y réjouis à Port-Saïd quand vous ou moi rossons Pasteur » (2 février 1862, BNF, naf 18111, fol. 72). De tels commentaires montrent que la controverse avait pris un tour dans lequel la célébrité et les ambitions personnelles avaient éclipsé les questions scientifiques. C'est sans doute la raison pour laquelle Pouchet prit, surtout en 1864, la

que retire chacun de partager les connaissances produites. 
peine de s'entourer des services de la presse. Il pouvait ainsi contrecarrer publiquement toute évaluation scientifique de ses expériences. Félix-Archimède Pouchet était en relation avec de nombreux journalistes, dont Noël, Chatin, Remusat, Meunier et Quesneville (Cantor-Coquidé, 1992). Tous ont oeuvré pour faire de la génération spontanée un débat public dans lequel les ressorts religieux, idéologiques et politiques furent, comme on sait, largement exploités.

Pouchet lui-même livrait aux journalistes les éléments qu'il souhaitait voir publier, et ne manquait pas, à l'occasion, d'en presser la parution. Il écrit ainsi à Noël: «Veuillez donc dire à ceux de vos amis chargés de ce soir que l'on se hâte ", ou bien: "Pour l'article du Journal de Rouen, vous m'obligeriez beaucoup s'il peut être publié rapidement [en rouge: je vous dirai pourquoi confidentiellement] »(BMR, ms $\mathrm{n}^{\circ}$ inventaire 1754). Dans l'action, il associe son ami journaliste à sa mission civilisatrice. Devenu « Pontife de l'hétérogénie », Félix Pouchet lance à Noël: « Sublime ami! recevez ma bénédiction » (ibidem). À la fin, le biologiste rouennais parait convaincu de l'équivalence des deux « forums » de la controverse, comme en atteste une lettre, du mois de juin 1864, adressée à Noël: « Par compensation, je pense que l'opinion publique est fortement pour nous, et l'on répète partout que l'infâme leçon de Paracelse II a fait un effet déplorable » (BMR, ms n inventaire 1754).

Une confiance aveugle dans le dogme de la génération spontanée, une quête avide de célébrité, une démission répétée devant les commissions chargées de décerner le prix Alhumbert et de juger les expériences sur la génération spontanée Pouchet: voici les raisons principales qui ont conduit Félix-Archimède Pouchet à l'échec. Ses travaux scientifiques n'ont peut être pas été jugés, mais son attitude de savant a sans doute été appréciée à sa juste mesure par les membres de l'Académie des Sciences. En attestent les derniers courriers de son vieil ami Flourens. En 1864, Pouchet a exigé qu'une commission soit instituée pour trancher entre ses expériences et celles de Pasteur. Le secrétaire perpétuel lui adresse deux lettres (MHR, FAP 3078).

Paris, le 25 février 1864.

Monsieur,

Vous avez désiré que vos expériences pussent être répétées devant une Commission de l'Académie. De son côté, M. Pasteur a bien voulu accéder à ce désir.

Une Commission a été nommée. 
Cette Commission vous invite à vous trouver à Paris pour procéder à ces expériences qui auront lieu du ler au 15 mars. Les frais qui pourraient être occasionnés, par suite de votre déplacement ou de vos expériences, seront au compte de l'Académie.

Veuillez agréer, Monsieur, l'assurance de ma considération très distinguée,

Flourens

La deuxième lettre de Flourens fait suite à la décision d'accorder à Pouchet un ultime report des expériences:

Paris, le 6 juin 1864.

Monsieur,

J'ai l'honneur de vous rappeler l'engagement que vous avez pris de vous trouver à Paris le 15 juin pour la répétition de vos expériences relatives à la génération spontanée.

Veuillez agréer, Monsieur, l'expression de ma considération très distinguée,

Flourens

Le ton de ces deux lettres est extrêmement sec. De plus, alors que les autres lettres de Flourens à Pouchet sont autographes ou comportent une finale de la main de l'auteur - du type: « considération très distinguée et toute mon affection » - seule la signature des deux lettres de 1864 est autographe. Flourens semble fatigué de voir son vieil ami se livrer à des exercices (affirmations dogmatiques, polémiques suivies de retraits, lobbying de la presse, recherche des honneurs) peu conformes avec une certaine idée de l'ethos scientifique.

\section{Conclusions}

La controverse sur la génération spontanée, prise en modèle par la sociologie des sciences d'obédience relativiste pour appuyer la thèse d'une détermination sociale des connaissances scientifiques, devrait être entièrement réécrite sur de nouvelles bases. La découverte d'un faux ouvre la voie à une réévaluation des rapports entre Pouchet et les membres de l'Académie des Sciences. Loin d'invalider une autonomie des connaissances scientifiques vis-à-vis du contexte religieux, idéologique ou politique, cette controverse montre au contraire que les réactions de l'Académie 
des Sciences étaient motivées. Elles le furent en partie sur des arguments scientifiques que les hétérogénistes ne parvinrent pas à retourner (Roll-Hansen, 1979; Gálvez, 1988). Elles le furent aussi sur une évaluation normative de l'attitude scientifique de Pouchet. De ce point de vue, c'est peutêtre sa collusion avec des instances extra-scientifiques qui lui valut une chute aussi brutale.

Si certains résumés de la controverse apparaissent aussi éloignés des conclusions auxquelles nous parvenons, c'est semble-t-il en raison de l'imbrication complexe de plusieurs facteurs. On peut en distinguer trois principaux:

1) C'est un effet pervers bien connu - qui affecte surtout les sciences sociales - que certains programmes de recherche peuvent se convertir en position doctrinale. Ses représentants tendent alors à sous-estimer les critiques pour se limiter au commentaire des propositions et des résultats compatibles avec les lignes directrices du programme de recherche. L'absence de discussion, dans certains compte-rendus récents, des arguments avancés par Roll-Hansen (1979), McMullin (1987), Gálvez (1988), Bunge (1992) ou Crosland (1992), témoigne malheureusement assez bien de cette tendance en sociologie des sciences.

2) Certains présupposés brouillent l'évaluation des phénomènes socio-historiques. Gálvez (1988: 356) montre que l'explication avancée par Farley et Geison (1974: 163-167), savoir que le jugement de la controverse aurait été biaisé par l'influence de Cuvier sur les membres de l'Académie des Sciences, ne résiste pas à l'analyse. Trois décennies — riches sur le plan scientifique séparent en effet la mort de Cuvier (1832) et le débat sur la génération spontanée (1859-1864). Mentionnons un autre présupposé du même type. Il est de coutume de regarder l'Institut comme un cénacle de gérontocrates. C'est ignorer les études précises sur l'âge moyen d'élection des membres de l'Académie des Sciences. Celui-ci a fortement varié dans le temps. Il était de 42 ans de 1800 à 1850, avant de passer à 50 ans en 1900 et à 65 ans en 1970 (Crosland, 1992: 101). Dans l'étude très documentée qu'il nous livre de cette institution, Grosland établit en outre la diversité des milieux d'origine et des opinions politiques de ses membres (1992: 177, 189). De tels présupposés, lorsqu'ils ne sont pas débusqués, nous préparent évidemment à accepter les conclusions relativistes.

3) Enfin, la plupart des interprétations relativistes de la controverse Pasteur-Pouchet sont fondées sur le compte rendu de Pennetier (1907). Ce fait est hautement indicatif, car Pennetier était un disciple direct de Pouchet. Codirecteur du Muséum d'Histoire naturelle de Rouen de 
1866 à 1872, il en deviendra le directeur à la mort de Pouchet (1872). L'étrange défense « sociologique » d'un Latour — qui écrit: « Les vainqueurs n'ont pas besoin d'être protégés par l'historien, mais seulement les vaincus, auxquels on donnera [...] une seconde chance devant le tribunal de l'histoire » (1989: 430) — devient ainsi un simple avatar de la version imaginée par Pouchet, et transmise par Pennetier, pour ne pas perdre la face. La critique que l'on peut adresser à ces interprétations est, en substance, identique à celle qu'on a jadis opposée à la biographie de Pasteur établie par Vallery-Radot (1900). René Vallery-Radot était, comme on sait, le propre gendre de Pasteur.

Toutes ces raisons font que la controverse entre Pasteur et Pouchet sur la génération spontanée, loin de pouvoir servir d'exemple emblématique du relativisme, est plutôt une pièce cohérente de sa critique. La réévaluation de ce débat scientifique sur la base de la correspondance de Pouchet avec les membres de l'Académie des Sciences rejoint les conclusions de Roll-Hansen (1979), McMullin (1987), Gálvez (1988), Bunge (1992), Crosland (1992) et Darmon (1995), et se rattache, par delà ces analyses de cas, à un programme rationaliste soutenu, entre autres, par Siegel (1987), Boudon et Clavelin (1994) et Koertge (1997).

\section{Bibliographie}

Barnes, S.B. et Dolby, R.G.A. (1970). The scientific ethos: A deviant viewpoint. European Fournal of Sociology, 11 : 3-25.

Ben-David, J. (1991). Scientific growth. Berkeley / Los Angeles / Oxford: University of California Press.

Boudon, R. et Clavelin, M. eds. (1994). Le relativisme est-il résistible? Regards sur la sociologie des sciences. Paris: Presses universitaires de France.

Bunge, M. (1991-1992). A critical examination of the new sociology of science. Philosophy of the Social Sciences, 21: 524560 et 22: 46-76.

Cantor-Coquidé, M. (1992). Félix-Archimède Pouchet, savant et vulgarisateur. Thèse de doctorat. Orsay: Université ParisSud.

Grellin, J.K. (1981). Pouchet. C.C. Gillispie, ed. Dictionary of scientific biography. New York: Charles Scribner's sons, 11: 109-110.

Crosland, M. (1992). Science under control. The French Academy of Sciences, 1795-1914. Cambridge: Cambridge University Press.

Farley, J. (1972). The spontaneous generation controversy (1700-1860): The origin of parasitic worms. Fournal of the History of Biology, 5: 95-125. 
Farley, J. et Geison, G. (1991). Le débat entre Pasteur et Pouchet: science, politique et génération spontanée au XIXe siècle en France. Callon, M. et Latour, B., La science telle qu'elle se fait. Paris: La Découverte: 87-145 [1974].

Gálvez, A. (1988). The role of the French Academy of Sciences in the clarification of the issue of spontaneous generation in the mid-nineteenth century. Annals of Science, 45: 345-365.

Geison, G. (1981). Pasteur. C.C. Gillispie, ed., Dictionary of scientific biography. New York: Charles Scribner's sons, 9: 350-414.

Isambert F.-A. (1985). Un « programme fort » en sociologie de la science? Revue française de Sociologie, 26: 481 -508.

Koertge, N., ed. (1997). A house built on sand: Exposing postmodernist myths about science. New York: Oxford University Press.

Lambert, P. (1936). Pasteur, ses précurseurs et ses adversaires. Livres, plaquettes rares, documents pour servir à l'histoire des sciences (catalogue XVII). Paris: Durtal.

Latour, B. (1989). Pasteur et Pouchet: hétérogenèse de l'histoire des sciences. M. Serres, Éléments d'histoire des sciences. Paris: Bordas, 423-445.

Laudan, L. (1990). Science and relativism. Chicago: University of Chicago Press.

Lécuyer, B.-P. (1989). La sociologie des sciences. L'Univers philosophique. Paris: Presses universitaires de France, 942948.

Lécuyer, B.-P. (1998). Sociologie de la connaissance et sociologie de la science. Kremer-Marietti, éd. Sociologie de la science. Bruxelles: Mardaga, 17-45.

Matalon, B. (1986). Sociologie des sciences et relativisme. Revue de synthèse, 4º série, 3: 267-290.

Mendelsohn, E. (1987). The political anatomy of controversy in the sciences. Engelhardt, H.T. et Caplan, A.L., eds. Scientific controversies. Case studies in the resolution and closure of disputes in science and technology. New York: Cambridge University Press, 93-124.

Merton, R.K. (1973). The sociology of science. Theoretical and empirical investigations. Edited with an introduction by Norman W. Storer. Chicago / London: The University of Chicago.

Norton, B. (1978). Karl Pearson and statistics: The social origins of scientific innovation, Social Studies of Science, 8: 334.

Pasteur, L. (1922). Oeuvres de Pasteur, réunies par Pasteur Vallery-Radot, tome II: Fermentations et générations dites spontanées. Paris: Masson.

Pasteur, L. (1951). Correspondance de Pasteur, réunie et annotée par Pasteur Vallery-Radot, tome II. La seconde étape: fermentations, générations spontanées, maladies de vins, des vers à soie, de la bière (1857-1877). Paris: Flammarion [1940].

Pennetier, G. (1907). Un débat scientifique. Pouchet et Pasteur. (Actes du Muséum d'Histoire naturelle de Rouen, tome XI). Rouen: Imprimerie J. Lecerf.

Pouchet, F.-A. (1853). Histoire des sciences naturelles au Moyen Âge, ou Albert le Grand et son époque considérés comme point de départ de l'école expérimentale. Paris: J.-B. Baillière.

Pouchet, F.-A. (1859). Hétérogénie, ou traité de la génération spontanée basé sur de nouvelles expériences. Paris: J.-B. Baillière et fils. 
Pouchet, F.-A. (1860). Genèse des proto-organismes dans l'air calciné et à l'aide de corps putrescibles portés à la température de 150 degrés, Comptes rendus de l'Académie des Sciences, 4 juin 1860, tome 1, 1014-1018.

Pouchet, F.-A. (1864). Nouvelles expériences de génération spontanée et la résistance vitale. Paris: J.-B. Baillière et fils.

Raynaud, D. (1998a). Les normes de la rationalité dans une controverse scientifique: l'exemple de l'optique médiévale. L'Année sociologique, 48 (2): 447-466.

Raynaud, D. (1998b). La controverse entre organicisme et vitalisme: étude de sociologie des sciences. Revue française de Sociologie, 34 (4): 73-102.

Roger, J. (1890). Les médecins normands du XIIe au XIXe siècle, tome I: Seine-Inférieure. Paris: G. Steinheil.

Roll-Hansen, N. (1979). Experimental method and spontaneous generation: The controversy between Pasteur and Pouchet. Fournal of the History of Medicine and Allied Sciences, 34: 273-292.

Siegel, H. (1987). Relativism refuted: A critique of contemporary epistemological relativism. Dordrecht: D. Reidel.

Vinck, D. (1995). Sociologie des sciences. Paris: Armand Colin.

Woolgar, S. (1988). Science: The very idea. London: Tavistock. 